\title{
Ererbte Transformation
}

Kommentar zu Matthias Bernt und Andrej Holm „Die Ostdeutschlandforschung muss das Wohnen in den Blick nehmen“
Matthias Bernt, Andrej Holm: ,Die Ostdeutschlandforschung muss das Wohnen in den Blick nehmen'

Kommentare von: Barbara Schönig, Carsten Keller, Carmen Leidereiter, Dieter Rink, Felix Ringel

Replik von:

Matthias Bernt, Andrej Holm

Im Februar 2020 war ich im Rahmen einer Gastdozentur an der University of California in San Diego. In einem meiner Vorträge dort diskutierte ich Prozess und Projekte der Internationalen Bauausstellung (IBA) StadtLand Thüringen (vgl. Doehler-Behzadi 2017) im Kontext des Wandels der Paradigmen räumlicher Planung sowie struktur- und gesellschaftspolitischer Peripherisierungsprozesse im Allgemeinen. Notwendigerweise war dabei auch über die spezifischen Auswirkungen dieser Politiken auf die durch die DDR und die Transformationsprozesse seit 1990 geprägte räumliche Entwicklung in Ostdeutschland zu sprechen. Mutmaßlich wird nur selten jemandem, der einen Vortrag über die in der Peripherisierungsforschung recht bekannte Stadt Pirmasens in der Südwestpfalz hält (vgl. Kühn 2016), unmittelbar eine Herkunft aus der Pfalz zugeschrieben.[1] Auch als Forscherin zur Transformation von Quartieren des sozialen Wohnungsbaus wurde meine Kritik an Verdrängung oder Marginalisierung nie als ein Hinweis auf biographische Wurzeln interpretiert. In der an diesen Vortrag anschließenden Diskussion aber wurde deutlich, dass mein US-amerikanischer Kollege mit großer Selbstverständlichkeit davon ausging, mit einer Wissenschaftlerin mit ostdeutschen Wurzeln zu sprechen. Aus deutscher Sicht erstaunt diese ganz selbstverständliche Zuschreibung nicht allein angesichts der noch immer erschreckend geringen Zahl an Professor_innen aus Ostdeutschland an deutschen Universitäten (vgl. Kollmorgen 2020). Sie ist auch inhaltlich überraschend: Denn mutmaßlich wäre das kaum passiert, hätte ich über die Stadt Pirmasens gesprochen, die übrigens meiner eigentlichen Heimat tatsächlich sehr nahe ist. Kritische Forschung zur räumlichen Entwicklung in Ostdeutschland schien offensichtlich eine biographische Verankerung nahezulegen.

Meine Erfahrung im akademischen Ausland spiegelt recht eindrücklich, was Matthias Bernt und Andrej Holm (Bernt/Holm 2020) in ihrem Text zum Ausgangspunkt ihrer Überlegungen machen. Die Auseinandersetzung mit räumlicher Entwicklung in Ostdeutschland ist in der Tat im wissenschaftlichen Feld marginal, und sie wird auch tendenziell einer qua Herkunft betroffenen Gruppe zugeschrieben. Demgegenüber konstatieren Bernt/Holm, 
dass die Forschung zu ostdeutschen Städten als solche in der Stadtforschung jenseits von empirischen Fallstudien als konzeptionell eigenständiges Feld keine Rolle spiele. Und dass umgekehrt die spezifische Verräumlichung des gesellschaftlichen Transformationsprozesses in der politik- oder sozialwissenschaftlichen Forschung wiederum heute nach einer großen Zahl an Publikationen Mitte der 1990er Jahre auch ein eher randständiges Feld sei. Folgerichtig schlagen sie vor, die Ostdeutschlandforschung stärker an die Stadtforschung und umgekehrt die Stadtforschung stärker an die Ostdeutschlandforschung anzubinden. Dabei sehen sie das Wohnen als ein Feld, das sich an der Schnittstelle zwischen gesellschaftlichen Verhältnissen und Alltagserfahrung befindet, und in dem sich wie in keinem anderen die tiefgreifende Wirkung der politischen, sozialen und ökonomischen Transformation spiegelt. Deshalb ist aus ihrer Sicht das Wohnen in besonderer Weise geeignet, Kenntnis über deren spezifische Strukturen und Wirkungen zu erlangen.

Bernt/Holm benennen mit ihrem Text in der Tat eine Fehlstelle in der Forschung sowohl der Stadtforschung als auch der Ostdeutschlandforschung selbst. Allerdings gälte es hierbei noch konzeptionelle Fragen zu klären. Schon die Frage, was und wer in einer auf das Wohnen gerichteten Forschung mit welchem Recht als ,ostdeutsch' zu betrachten wäre - etwa qua Geburt, qua familiäre Wurzeln, qua Wohnort -, scheint nicht trivial. Ebenso gilt es eine Haltung zu der Frage zu entwickeln, ob und inwieweit Forschung, die nach Differenzen in einem vielfach verwobenen Feld gesellschaftlicher und räumlicher Entwicklung in Deutschland sucht, gerade diese nicht auch stetig neu verfestigt und dadurch die Pluralität ostdeutscher Entwicklungen und Erfahrungen und gegebenenfalls auch Parallelitäten zu Westdeutschland überschreibt. Eine wissenschaftliche Konzeptionalisierung einer Forschung zu Ostdeutschland muss diese Fragen sicher klären, was dieser Kommentar noch nicht leisten kann.

Stattdessen will ich hier die Idee einer spezifisch auf Ostdeutschland bezogenen Wohnungsforschung weiterspinnen und andeuten, wie sich diese „als historisch konkrete Entfaltung ökonomischer und politischer Machtverhältnisse“ (Bernt/Holm 2020: 110) im Kontext allgemeiner wirkender Transformationsprozesse von Stadtentwicklung und Wohnungsversorgung interpretieren ließen. Dabei richte ich meine Perspektive hier ganz schlicht auf Wohnen und Stadtentwicklung im geographisch bestimmbaren Raum Ostdeutschlands. Aus Sicht der Planungswissenschaften erscheint allerdings zunächst die Identifikation einer Fehlstelle irritierend. Denn tatsächlich findet sich eine Vielzahl an mindestens auch wohnungsbezogenen und vielfach aufschlussreichen empirischen Arbeiten zu Stadterneuerung in Innenstädten und Großwohnsiedlungen (1990er), Leerstand und ,Stadtumbau Ost', also Abriss (seit den frühen 200oern), energetischer Ertüchtigung und altersgerechter Erneuerung (seit 2000ern) sowie jüngst zur Segregation und Gentrifizierung auch in ostdeutschen Städten. Im Sinne des von Bernt/ Holm geforderten Wissens und Verständnisses der politisch-institutionellen Bedingungen des Transformationsprozesses aber dürften viele dieser Arbeiten wohl eher als Quellen hilfreich sein als dass sie diesen selbst zum Thema machten.

Will man sich nun aber dieser Aufgabe widmen, so verdient jenseits der unmittelbar auf die Wohnung bezogenen Aspekte die bei Bernt/Holm 
angedeutete Wechselwirkung der Transformation der Wohnung vom Gebrauchsgut zur Ware mit der Entwicklung der Städte in Ostdeutschland eine präzisere Betrachtung. Denn mit dieser Transformation wurden nicht nur Unsicherheiten bezüglich der eigenen Wohnung für viele zum Alltag. Auch das Wohnumfeld, also Quartier und Stadt, veränderte sich mit dem Wandel der Logiken von Wohnraumbewirtschaftung sowie Stadtentwicklung und den darin involvierten Akteuren (vgl. Häußermann 1996: 302-306). Dies gilt in besonderem Maße für die kommunale Planung (vgl. Betker 2005: Teil IV). Nicht staatliche Leitlinien und planwirtschaftliche Vorgaben - oft mit einem Fokus auf Wohnungsproduktion und Infrastruktur - bestimmten Stadtentwicklungsplanung nach 1990. Die Kommunen traten ein in einen Aushandlungsprozess um Stadtentwicklung und einen Wettbewerb um Menschen, Investitionen, Entwicklungen oder auch Fördermittel, der mit zunehmender Desinvestition, einem Anstieg der Arbeitslosigkeit und der Schrumpfung beziehungsweise Alterung der Bevölkerung dringlicher wurde und regionalräumlichen Polarisierungen Vorschub leistete. Dieser Umbruch in der Stadtentwicklung erfolgte in einer neuen Administrationsstruktur, insbesondere in den frühen 1990er Jahren zum Teil vor dem Hintergrund fehlender planungsrechtlicher Regulierungen und vielfach mit neuem Personal, oft aus dem Westen (vgl. Betker 2005: 242-3), wobei dieses Feld seinerseits noch der Forschung bedürfte. Es dürfte allerdings kaum zu bestreiten sein, dass die westdeutsch sozialisierten Planer_innen ein im Westen längst hegemoniales kulturelles Missbehagen gegenüber industriellem Wohnungsbau und damit den Plattenbausiedlungen in die Planungsbehörden in Ostdeutschland einbrachten. Begleitet vom Narrativ der Misswirtschaft der hoch verschuldeten DDR-Wohnungswirtschaft, in das die von Bernt/Holm beschriebene Altschulden-Problematik bald umgedeutet wurde, und in Ansehung ihrer tatsächlichen (städte)baulichen Mängel waren die Großwohnsiedlungen diskreditiert. Im Vergleich zu den zwar sorgebedürftigen, aber oft schnell privatisierten oder restituierten innerstädtischen Altbauten einerseits und den in der BRD zwar professionell missliebigen, aber politisch opportunen und daher vielerorts rasch ausgewiesenen Einfamilienhausgebieten andererseits wurden die Großsiedlungen trotz ihrer anfänglichen gesellschaftlichen Durchmischung und umfänglicher Investitionen schon Ende der 1990er Jahre sukzessive zum ostdeutschen Prototyp ,überforderter Nachbarschaften“ - was nicht zuletzt durch eine „kulturelle Entwertung durch die Medien“ verstärkt wurde (vgl. GDW 1998: 192). In kurzer Zeit wurde letztlich die gesamte zuvor auch staatlich sanktionierte Idee von Stadt, und mit ihr die kulturelle Bewertung ihrer unterschiedlichen Stadtteile, von außen nach innen gekehrt. Wie Steffen Mau eindrücklich beschreibt, wurde plötzlich aus dem fortschrittlichen Quartier der Werktätigen der sozialistischen Gesellschaft ein Ort, in dem jene zu verblieben schienen, die in der ,neuen Gesellschaft ökonomisch und gesellschaftlich nur schwer ankamen und/oder jedenfalls in ihr sprichwörtlich keinen neuen Raum finden konnten oder wollten (vgl. Mau 2019: 42, 158-9): alte SED-Anhänger_innen, Arbeitslose und später Migrant_innen, die keine Alternative auf dem Wohnungsmarkt hatten.

Was sich hier als Transformation der Wohnungsversorgung in Ostdeutschland darstellt, ist tatsächlich mit Entwicklungen in Westdeutschland aufs Engste verbunden. Das gilt einerseits wissenschaftsgeschichtlich: Denn das 
,Wohnen' als solches wurde fast 30 Jahre lang nicht nur im Osten, sondern auch im Westen wenig bis gar nicht beforscht (Schipper/Schönig 2021). Es war in seiner ganzen Komplexität als soziale Praxis, als Gegenstand staatlicher Steuerung und Regulierung, als baulich-räumliche Materialität und Ergebnis sozialer Aushandlungsprozesse (Schönig/Vollmer 2020) nicht nur politisch, sondern auch für die Stadtforschung kaum Thema.

Andererseits sind auch die Entwicklungen von Wohnen und Stadtentwicklung selbst verwoben (vgl. Schönig 2020a, 2020b): Das gilt erstens für die Diskreditierung der Bestände des industriellen Wohnungsbaus, die auch in Westdeutschland Gegenstand der Kritik aus ästhetisch-kultureller und ökonomischer Sicht waren (vgl. Zupan 2018). Dabei war die Durchsetzung dieser Perspektiven keineswegs nur ein Transfer von Planungsparadigmen von West nach Ost, sondern sie knüpfte an eine verbreitete Kritik an der baulichen Qualität der Großwohnsiedlungen, der Vernachlässigung der Innenstädte und der Normierung des Wohnens an, wie sie sich beispielsweise literarisch schon 1974 im Romanfragment Franziska Linkerhand von Brigitte Reimann findet und auch von vielen Planer_innen und Stadtsoziolog_innen geteilt wurde (vgl. Hannemann 1996: 118, 122).

Zweitens vollzog sich ab den 1990er Jahren ein tiefgreifender Wandel von Stadtentwicklungspolitik in West wie Ost, wo sich Kommunen von der Vorstellung lokaler Wohlfahrtspolitik einerseits und sozialistisch-planwirtschaftlicher Stadtentwicklung andererseits verabschiedeten. Unter dem Druck von Strukturwandel und kommunalen Finanzkrisen fokussierten sie die Stadtentwicklung in ähnlicher Weise auf Unternehmen, Mittelschichten und Fiskalpolitik. Die lokale Wohnungsversorgung prägte dies erheblich, zum Beispiel hinsichtlich der Ausweisung von Neubauflächen, des Verkaufs kommunaler Liegenschaften oder der Aufwertung von Innenstädten.

Drittens erfolgte das schlussendlich im Einklang mit der ohnedies durch die Einigungspolitik und das Altschuldenhilfegesetz vorgesehenen schnellstmöglichen Anpassung des Wohnungsmarkts im Osten an jenen des Westens (vgl. Borst 1997), der seinerseits in Bewegung war: Die neuen Bundesländer traten in eine BRD ein, in der das Wohnungsgemeinnützigkeitsgesetz gerade abgeschafft worden war, der national geförderte soziale Wohnungsbau als Auslaufmodell galt und die Verantwortung für soziale Wohnungsversorgung sukzessive auf Kommunen übertragen wurde. So wurde folgerichtig beispielsweise über eine Überführung der vormals staatlichen Wohnungsunternehmen in eine gemeinnützige Wohnungswirtschaft von Anfang an gar nicht mehr nachgedacht.

Was sich in den hiermit beschriebenen Prozessen der Transformation des Wohnens in Ostdeutschland zeigt, ist in der Tat nicht zu deuten als eine ,nachholende Modernisierung'. Vielmehr handelt es sich um Prozesse der Transformation von Wohnungsversorgung und Stadtentwicklungspolitik, die in Ost- wie Westdeutschland - ebenso wie auch in anderen westlichen Wohlfahrtsstaaten - gleichzeitig liberalisiert und kommodizifiert wurden. Dabei bildeten sich in Abhängigkeit von den lokalen Rahmenbedingungen und Akteurskonstellationen durchaus lokal geprägte Regime der Wohnungsversorgung und Stadtentwicklung heraus, die sich keineswegs nur zwischen West und Ost unterscheiden (Rink/Egner 2020). So ist zwar mit großer Sicherheit davon auszugehen, dass sich Städte wie Suhl und Altenburg im 
strukturschwachen Raum Thüringens (vgl. Schönig 2020a) ähnlicher sind als Suhl und Pirmasens. Aber ohne Frage unterscheiden sich Suhl und Jena hinsichtlich ihrer jeweiligen,Wohnungsfragen' mehr als Jena und Frankfurt am Main.

All dies aber spricht nicht dagegen, Wohnen und Stadtentwicklung in Ostdeutschland als eigenständiges Feld der Forschung zu etablieren. Im Gegenteil: In Ostdeutschland erfolgte die hier beschriebene Transformation in einem deutlich rasanteren Tempo (vgl. Häußermann 1996: 302-6). Sie ging aus von einer verstaatlichten Raumordnungspolitik und einer nahezu vollständigen Dekommodifizierung des Wohnungsmarkts und startete mit einem großen Bestand sanierungsbedürftiger Gebäude. Sie vollzog sich parallel zu einem individuell und gesellschaftlich tiefgreifenden Einschnitt in das soziale Leben und die Erwerbsbiographien, der die mit der Kommodifizierung des Wohnraums einhergehenden Unsicherheiten für Mieter_innen umso spürbarer werden ließ, aber auch Quartiere und Städte sichtbar veränderte. Und zugleich schuf der von Bernt/Holm angesprochene Transfer von staatseigenem, Volksvermögen' nicht nur an Boden und Immobilien, sondern vor allem auch an Industrie in den Westen (Böick 2018; Mau 2019: 177) eine dauerhafte - eben verräumlichte - Basis für die Ungleichverteilung der Vermögen zwischen Ost und West.[2] In der Folge all dessen sind die Spuren nicht nur der DDR, sondern auch des Transformationsprozesses seit 1990 noch heute in den individuellen Biographien, aber auch in Stadt- und Regionalentwicklung, sicht- und spürbar - und werden es auch noch eine Weile bleiben. Und auch die Struktur der Wohnungsversorgung im Osten unterscheidet sich deutlich von jener in Westdeutschland: Die Eigentümerquote ist niedriger, der Anteil genossenschaftlicher und kommunaler Bestände gerade in kleineren Kommunen größer, Leerstandsquoten und Erneuerungsbedarfe sind höher (Schönig 2020b).

Schon dieser skizzenhafte Abriss zeigt: Der Fokus auf die Transformation des Wohnens in Ostdeutschland eröffnet die Möglichkeit zu verstehen, wie die auch andernorts wirkenden Transformationen sich unter anderen (historischen, gesellschaftlichen, politisch-institutionellen, ökonomischen) Rahmenbedingungen vollzogen und auch durchaus differente gesellschaftliche und (sozial)räumliche Prozesse in Gang setzten. Zusätzlich erweist sich der Fokus auf das Wohnen auch deshalb als produktiv, weil er den Blick auf die ebenfalls durchaus spezifischen Effekte anderer Felder gesellschaftlicher Restrukturierung lenkt: Wohnungsleerstand und -investitionsbedarf in strukturschwachen Regionen erzählen auch davon, wie die wettbewerbsorientierte Strukturpolitik aufgrund des radikalen Umbaus der DDR-Industrielandschaft nach 1990 Peripherisierungsprozesse intensivierte und zu besonders tiefen „Frakturen“ (Mau 2019: 13) in einigen ostdeutschen Regionen führte.

Nun sind auch in anderen postsozialistischen Staaten die Spuren der Transformation noch sichtbar. Doch die auch kartographisch immer wieder eindrücklich darstellbare ungleiche Verteilung von Rente, Wachstum, Reichtum, Bildung, Excellenzclustern und Sonderforschungsbereichen, Fußball-Bundesligaclubs und vielem anderem mehr innerhalb Deutschlands verdeutlicht allzu eindrücklich die längst nicht ver(sch)wundene deutsch-deutsche Trennung beziehungsweise Einigung. Und sie bietet eben immer wieder auch einen Nährboden für öffentliche Diskurse um historische Ungerechtigkeit einerseits oder mangelnde Leistungsfähigkeit und Undankbarkeit andererseits. 
Gerade im Lichte dieser Beobachtungen zeigt sich aus meiner Sicht der Wert der subjektbezogenen, biografisch verankerten Arbeiten, die Bernt/ Holm als dritte und aktuelle Phase der Ostdeutschlandforschung identifizieren (Bernt/Holm 2020: 100 f.). Sie gewähren Einblick in die Dramatik der „Implosion“ (Mau 2019: 113) der sozialen Ordnung der DDR innerhalb kürzester Zeit für die ostdeutsche Gesellschaft und die Biografie jedes/r Einzelnen. Ein Einblick, den ich angesichts der offensichtlich zwischen Ost und West vielfach divergierenden Erfahrungen auch als Westdeutsche als notwendige Voraussetzung für die von Bernt/Holm geforderte Aufklärung und Wissensvermittlung zur Alltagserfahrung und den Verfahren und Prozessen der Transformation erachte. Denn diese machen die Subjektkonstruktionen in Ostdeutschland zu einem für den Diskurs insgesamt verfügbaren Wissen. Dass hierbei über die Deutung und Hegemonie der Narrative zu streiten sein wird, steht außer Frage.

Anknüpfend an einen solchen subjektbezogenen, aber auch auf den schon vorhandenen empirischen Wissensbestand und die wissenschaftlichen Diskurse um Stadtentwicklung und Wohnungsversorgung, sollte die Transformation des Wohnens in Ostdeutschland vor dem Hintergrund ihrer spezifischen politisch-institutionellen und ökonomischen Bedingungen betrachtet werden. Denn in der Tat wirkt diese Transformation - im Sinne der zweiten konzeptionellen Forderung von Bernt/Holm - als „Rahmenbedingung der aktuellen Entwicklungen“ (Bernt/Holm 2020: 110) fort. Sie stellt gewissermaßen eine ererbte Transformation dar, deren Folgen das Wohnen, und nicht zuletzt auch dadurch Alltagshandeln ebenso wie Stadtentwicklung prägen und weiterhin prägen werden.

Die Bauhaus-Universität Weimar unterstützt die Publikation dieses Beitrags durch eine institutionelle Vereinbarung zur Finanzierung von Publikationsgebühren.

\section{Endnoten}

[1] Pirmasens ließe sich ganz grob vielleicht als eine Art ,westdeutsches Suhl' bezeichnen: demographisch stark schrumpfend, gezeichnet vom Niedergang der lokal dominanten Industrie (Schuhproduktion), ebenfalls umgeben von Wald, aber noch stärker als Suhl abseits großer Zentren und Verkehrswege und zugleich im deutsch-französischen Grenzgebiet gelegen.

[2] Führt man sich vor Augen, dass im gegenwärtigen finanzialisierten Kapitalismus Vermögenszuwächse mittlerweile vor allem durch Immobilienkapital erwirtschaftet werden und dieses eben vor allem im Westen konzentriert ist (Baldenius/Kohl/ Schularick 2019: 21), wird deutlich, dass sich diese Ungleichheit nicht in den nächsten Generationen erübrigen wird.

\section{Autor innen}

Barbara Schönig befasst sich in Lehre und Forschung mit Prozessen und Akteuren der Planung und Produktion von Stadt und Raum. Ihre Forschungsfelder sind interdisziplinäre Wohnungsforschung, sozialer Wohnungsbau, Partizipation und Stadtentwicklung sowie die 
(Re-)Strukturierung städtischer, suburbaner ebenso wie ländlicher Räume im Kontext gesellschaftlicher Transformation.

barbara.schoenig@uni-weimar.de

\section{Literatur}

Baldenius, Till / Kohl, Sebastian / Schularick, Moritz (2019): Die neue Wohnungsfrage. Gewinner und Verlierer des deutschen Immobilienbooms. Bonn: Macrofinancelab, Universität Bonn.

Bernt, Matthias / Holm, Andrej (2020): Die Ostdeutschlandforschung muss das Wohnen in den Blick nehmen. Plädoyer für eine neue politisch-institutionelle Perspektive auf ostdeutsche Städte. In: sub\urban. zeitschrift für kritische stadtforschung 8/3, 97-114.

Betker, Frank (2005): „Einsicht in die Notwendigkeit.“ Kommunale Stadtplanung in der DDR und nach der Wende (1945-1994). Stuttgart: Steiner.

Böick, Marcus (2018): Die Treuhand. Ideen - Praxis - Erfahrung: 1990-1994. Bonn: Bundeszentrale für politische Bildung.

Borst, Renate (1997): Verfahren und Wirkungen der Privatisierung von Mietwohnungen in den neuen Bundesländern seit 1990. In: Uta Schäfer (Hg.), Städtische Strukturen im Wandel. Opladen: Leske + Budrich, 117-216.

Bundesverband Deutscher Wohnungsunternehmen e.V. (GDW) (Hg.) (1998): Überforderte Nachbarschaften. Zwei sozialwissenschaftliche Studien über Wohnquartiere in den alten und den neuen Bundesländern. Köln: GDW.

Doehler-Behzadi, Marta (2017): Wie die IBA Thüringen Stadtland beschreibt. In: Arch+ 228, Stadtland - Der neue Rurbanismus, 12-19.

Hannemann, Christine (1996). Die Platte: Industrialisierter Wohnungsbau in der DDR. Braunschweig: Vieweg.

Häußermann, Hartmut (1996): Die Transformation des Wohnungswesens. In: Wendelin Strubelt / Annette Becker (Hg.), Städte und Regionen - räumliche Folgen des Transformationsprozesses. Berichte der Kommission für die Erforschung des Sozialen und Politischen Wandels in den Neuen Bundesländern e.V. (KSPW) Opladen: Leske + Budrich, 289-325.

Kollmorgen, Raj (2020): Zwischen Austausch und Marginalisierung: Ostdeutsche in den Eliten nach der Vereinigung. In: BpB (Hg.), Dossier: Lange Wege der Deutschen Einheit. https://www.bpb.de/geschichte/deutsche-einheit/lange-wege-der-deutscheneinheit/310065/ostdeutsche-in-den-eliten (letzter Zugriff am 18.09.2020).

Kühn, Manfred (2016): Peripherisierung und Stadt. Städtische Planungspolitiken gegen den Abstieg. Bielefeld: transcript.

Mau, Steffen (2019): Lütten Klein. Leben in der ostdeutschen Transformationsgesellschaft. Berlin: Suhrkamp.

Rink, Dieter / Egner, Björn (Hg.) (2020): Lokale Wohnungspolitik. Beispiele aus deutschen Städten. Baden-Baden: Nomos.

Schipper, Sebastian / Schönig, Barbara (2021): Die ewig neue Wohnungsfrage! Auf den Spuren bundesdeutscher Debatten zur sozialen Wohnraumversorgung. In: Björn Egner / Stephan Grohs (Hg.), Die Rückkehr der Wohnungsfrage. Ansätze und Herausforderungen lokaler Politik. Wiesbaden: Springer VS, im Ersch.

Schönig, Barbara (2020a): Paradigm Shifts in Social Housing After Welfare-State Transformation: Learning from the German Experience. In: International Journal of Urban and Regional Research, 16.6.2020.

Schönig, Barbara (2020b): Unter dem Radar. Wohnungsfragen abseits der Wachstumsräume in Thüringer Klein- und Mittelstädten. In: Barbara Schönig / Lisa Vollmer (Hg.), Wohnungsfragen ohne Ende?! Ressourcen für eine soziale Wohnraumversorgung. Bielefeld: transcript, 207-227.

Schönig, Barbara / Vollmer, Lisa (2020): Wohnungsfrage(n) ohne Ende und überall?! Sechs Thesen für eine interdisziplinäre Wohnungsforschung. In: Barbara Schönig / Lisa Vollmer (Hg.), Wohnungsfragen ohne Ende?! Ressourcen für eine soziale Wohnraumversorgung. Bielefeld: transcript, 7-33.

Zupan, Daniela (2020): Leitbildwechsel. Dynamiken und Charakteristika städtebaulicher Innovationsprozesse. Detmold: Verlag Dorothea Rohn. 
